\title{
Editorial
}

\section{Candan Tamerler*}

Mechanical Engineering Department and Institute of Bioengineering

Research, University of Kansas, Lawrence, KS, USA

This themed issue of Surface Innovations covers a major interest area, bio-nano interfaces and their engineering applications. Bio-nano interfaces are the critical components of bio-related materials, processes and devices covering a wide range of areas from engineering to life sciences. In biological or biomimetic systems, soft interlayers composed of biological components or polymers define spatial boundaries and different phases. Understanding the nature of interactions at these soft interlayers and utilizing this information for engineering hybrid materials and systems is attracting an increasing amount of interest since the application areas span a wide range from biomedicine to smart textiles. The current issue features six contributions that are collection of original research and review papers on the selected topics chosen to represent different application areas.

Yamashita ${ }^{1}$ presents a comprehensive review on biotemplating fabrication approaches to produce nanoscale materials towards next-generation devices. Generating sub-10 nm size structures has been challenging using conventional miniaturization techniques; biotemplated synthesis of inorganic materials utilizes the nanometer size precision that is offered by proteins. The review describes the use of cage-protein systems, such as ferritin for nanoscale materials synthesis, for the purposes of device fabrication. Examples include floating gate memories produced using biotemplated nanoparticles, dye-sensitized solar cells using bioconjugates of carbon nanotubes and DNA binding proteins for thermoelectric devices.

VanOosten et $a l^{2}$ provide a biomolecular self-assembly approach to coat the silver nanoparticles to modulate the biological effect. Despite the promising antimicrobial property of silver, there is still some concern on the potential cytotoxicity of silver nanoparticles. The authors investigate the effect of the biological surface functionalization technique as a way to control antimicrobial properties and prevent potential cytotoxicity of the nanoparticles. Engineered peptide-based biomimetic interface created on the silver nanoparticles demonstrates a significant reduction in cytotoxicity with retained antimicrobial property.

Despite the critical importance, there is limited information on the corrosion properties of metallic implants in the biological environment. Drelich et $a l^{3}$ demonstrate that different surface engineering of thin oxide films on zinc implants leads to tunable rates of biocorrosion. Their analysis on degradation rate for zinc implants shows that degradation rate depends heavily on the quality and the stability of the oxide films. Their findings provide a perspective towards designing smart biodegradable stents with desired responsive behavior.

Kilic and $\mathrm{Kok}^{4}$ provide a review on design of biomimetic lipid bilayer platforms to study biological membranes and their constituent lipid and proteins. The article summarizes the formation of solid-supported lipid bilayers as hydrophilic or hybrid bilayers, the available characterization techniques to investigate layer formation processes, and it describes the robustness of the layers and provides examples on their utilization including studying different biomolecular or cell-cell or cell-matrix interactions.

Dickinson et $a l .{ }^{5}$ describe a novel peeling test to study interfacial interactions in a graphene oxide and polymer nanocomposite. This technique allows the study of different matrix-filler interactions in developing novel nanocomposites. By creating multiple graphene oxide nanosheets and a variety of industrial polymer combinations, the authors study the interfacial interactions between the hybrid layers. An affinity-based ranking for the individual combinations is provided by investigating the effects of thermal reduction of graphene oxide nanofillers on interfacial adhesion.

Porous nanofibers demonstrate superior activity compared to smooth surfaces in diverse application ranging from protein adsorption to electrical conductivity. Illangakoon et $a l^{6}{ }^{6}$ presents a pressurized gyration method, which builds upon simultaneous utilization of centrifugal spinning and solution blowing to produce porous nanofibers ranging from smooth to rough. The proposed method provides different surface morphologies that could find applications in a wide range of areas from biomedicine to textile engineering.

We hope that you will enjoy the issue and find it relevant to your interests on surface innovations.

\section{REFERENCES}

1. Yamashita I (2016) Biological path for functional nanostructure fabrication and nanodevices. Surface Innovations 4(3): 111-120, http://dx.doi.org/10.1680.jsuin.16.00015.

2. VanOosten SV, Yuca E, Karaca BT et al. (2016) Biosilver nanoparticle interface offers improved cell viability. Surface Innovations 4(3): 121-132, http://dx.doi.org/10.1680.jsuin.16. 00010 . 
3. Drelich AJ, Bowen PK, LaLonde L, Goldman J and Drelich JW (2016) Importance of oxide film in endovascular biodegradable zinc stents. Surface Innovations 4(3): 133-140, http://dx.doi. org/10.1680.jsuin.16.00014.

4. Kilic A and Kok FN (2016) Biomimetic lipid bilayers on solid surfaces: models for biological interactions. Surface Innovations 4(3): 141-157, http://dx.doi.org/10.1680.jsuin.16. 00008 .
5. Dickinson LR, Kranbuehl DE and Schniepp HC (2016) Assessing graphene oxide/polymer interfacial interactions by way of peeling test. Surface Innovations 4(3): 158-166, http://dx.doi.org/10.1680.jsuin.16.00009.

6. Illangakoon UE, Mahalingam S, Colombo P and Edirisinghe $M$ (2016) Tailoring the surface of polymeric nanofibres generated by pressurised gyration. Surface Innovations 4(3): 167-178, http://dx.doi.org/10.1680.jsuin.16.00007. 